\title{
Is subjective word familiarity a meter of ambient language? A natural experiment on effects of perceptual experience
}

\author{
EDWARD T. AUER, JR., LYNNE E. BERNSTEIN, and PAULA E. TUCKER \\ House Ear Institute, Los Angeles, California
}

\begin{abstract}
The present study examined the sensitivity of a subjective familiarity measure to differences in word exposure within and between populations that differ dramatically in their perceptual experience. Descriptive measures of language ability and subjective familiarity ratings for 450 words were collected from a group of college-educated adults with normal hearing and a group of college-educated deaf adults. The results demonstrate the sensitivity of subjective familiarity ratings to both between- and within-group differences in word experience. Specifically, the deaf participants consistently rated words as less familiar than did hearing participants. Furthermore, item-level correlations within a participant group were higher than ones between groups. Within groups, mean familiarity ratings were correlated with descriptive measures of language ability. The results are discussed in relation to a simple sampling model of word experience and the language experience of the participant groups.
\end{abstract}

Humans store and use the statistical properties of their surrounding linguistic environment. For example, adults have demonstrated a sensitivity to statistical properties, such as frequency of phonetic patterns in the speech stream (Pitt \& McQueen, 1998; Vitevitch, Luce, Charles-Luce, \& Kemmerer, 1997), experience with words (Amano, Kondo, \& Kakehi, 1995; Gaygen \& Luce, 1998), meaning usage for lexically ambiguous words (Seidenberg, 1997), and co-occurrence among words (Seidenberg, 1997). Word frequency of occurrence, in particular, has received substantial attention over the past several decades (e.g., Balota \& Chumbley, 1984; Howes, 1957; Luce \& Pisoni, 1998; Plaut, McClelland, Seidenberg, \& Patterson, 1996; Savin, 1963; Seidenberg \& McClelland, 1989). Word frequency is a potent predictor of the speed and ease of both spoken and printed word recognition, with recognition being facilitated for more frequent words (see Connine, Mullennix, Shernoff, \& Yelen, 1990; Forster, 1976; Howes, 1957; Luce \& Pisoni, 1998; Morton, 1969; and Savin, 1963, among others). The present study examined a subjective word frequency measure, familiarity, within and between populations that differ dramatically in their perceptual experience - in this case, deaf ${ }^{1}$ versus hearing college-educated adults.

This work was supported by Research Grant R01 DC02107 from the National Institute on Deafness and Other Communication Disorders, National Institutes of Health. The authors thank David B. Pisoni for making available the stimulus list used in the familiarity task and the familiarity ratings from the Hoosier Mental Lexicon. The authors also thank Brian Chaney, Sheri Hithe, and two anonymous reviewers for their helpful advice and assistance. A file containing the item mean familiarity data is available at http://www.hei.org/htm/eauer.htm. Correspondence should be addressed to E. T. Auer, Jr., Spoken Language Processes Laboratory, House Ear Institute, 2100 West Third St., Los Angeles, CA 90057 (e-mail: eauer@hei.org).

\section{Subjective Word Familiarity}

Objective word frequency counts based on text corpora (Carroll, Davies, \& Richman, 1971; Kučera \& Francis, 1967; Thorndike \& Lorge, 1944) have been used as estimates of experienced word exposure. The method is not, however, without its detractors. Gernsbacher (1984) has pointed out that because objective frequency counts are dependent on the text corpus used, various estimation problems can result (see also Carroll, 1970). Gernsbacher (1984) proposed subjectively rated familiarity as a substitute for objective frequency counts (see also Amano et al., 1995; Carroll, 1971; Gaygen \& Luce, 1998; Gilhooly \& Logie, 1980; Nusbaum, Pisoni, \& Davis, 1984; Rubin, 1976). On the basis of a series of printed word recognition experiments, she concluded that subjective familiarity was a more accurate index of word experience than objective counts of words in texts. On the other hand, although subjective familiarity is an effective predictor of word recognition, substantial debate exists regarding its purity as an estimate of word exposure rate. Because word familiarity ratings are subjective off-line measures, individuals could base their ratings on several different sources of knowledge, including memory for modality-specific exposure (Amano et al., 1995; Brown \& Watson, 1987; Connine et al., 1990; Garber \& Pisoni, 1991; Gaygen \& Luce, 1998), phonotactics (Eukel, 1980; Landauer \& Streeter, 1973), concreteness of meaning (Balota, 1994; Balota, Ferraro, \& Connor 1991), concept familiarity (Hirsh \& Funnell, 1995), number of meanings (Balota et al., 1991), and age of acquisition (Brown \& Watson, 1987).

General agreement does exist that rates of word exposure are related to subjective familiarity. If this is true, familiarity ratings ought to be sensitive to population and individual differences in word exposure (see Gernsbacher, 
1984). This fundamental presumption has not, however, received adequate testing. The literature offers two moderately supportive studies.

Carroll (1971) obtained evidence that subjective word frequency estimates were more reliable among lexicographers than individuals drawn from the general collegeeducated adult population. However, he did not test for significant differences between the two groups. Lewellen, Goldinger, Pisoni, and Greene (1993) indirectly provide evidence that familiarity ratings are sensitive to differences in word knowledge across groups of highversus low-verbal college students. Normal-hearing undergraduates were screened and then grouped into those who consistently scored either high or low on multiple measures of language experience, including word familiarity. They were then tested on three lexical processing tasks with printed word stimuli: naming, lexical decision, and semantic categorization. The pattern of results in the lexical processing tasks supported the conclusion that individuals with greater language experience, including higher mean word familiarity, are more efficient at lexical processing. Unfortunately, it is not known whether familiarity ratings alone would have resulted in significantly different groups.

In the present study, the hypothesis that subjective word familiarity sensitively measures differences in word exposure was directly tested with two groups of native English speakers. In one group, individuals had reduced and possibly delayed ${ }^{2}$ exposure to ambient language due to early-onset severe to profound hearing impairment (deaf). The other group was composed of typical undergraduates.

\section{Deafness and Lexical Knowledge}

The age at which a child's hearing loss occurs is often determinative for educational placement, mode of communication, and language proficiency and experience. Difficulty in acquiring a spoken language is correlated with degree of hearing impairment in children with congenital or prelingual impairments. Academic achievement levels of deaf children as a population, are significantly below those of their hearing cohort. Mean reading comprehension scores for deaf children plateau at approximately a third-grade level (Allen, 1986). Only 10\% of all deaf 18-year-olds can read at or about the eighth grade level (Trybus \& Karchmer, 1977).

Approximately $90 \%$ of children with profound hearing impairments are born into families with hearing parents and a spoken language as the basis for communication (Schein, 1989). Nevertheless, congenitally or prelingually deaf children are likely to use some form of manual communication as their preferred communication mode and/ or as a supplement to lipreading. Manual communication can include a form of English-based signing (signs presented in English syntax and word order; Akamatsu \& Stewart, 1998); American Sign Language (ASL), a manual language distinct from English; or Cued Speech, a method in which eight different handshapes at specific placements around the mouth are used to disambiguate like-appearing speech segments (Cornett, 1967). Approximately $10 \%$ of severely and profoundly deaf children attend oral schools, where they use speech, lipreading, and residual hearing to communicate (Allen, 1994). Typically, all deaf students also receive speech production and lipreading training, with varying degrees of success.

In the present study, a specific subset of deaf adults was chosen because of their lifelong hearing impairment and because they have mastered English adequately to gain college admission. Their English achievement is most likely the result of reading, lipreading, ${ }^{3}$ and/or the use of some type of an English-based sign system. Later language acquisition in this group likely affected not only total quantity of word exposure but also its distribution.

Relatively few studies have attempted to assess in detail the specific vocabulary knowledge of deaf individuals (cf. Walter, 1978b), and the focus in these studies has been primarily on children and early adolescents (Griswold \& Commings, 1974; Silverman-Dresner \& Guilfoyle, 1972; Walter, 1978a). Overall, vocabulary knowledge is poor in those with congenital or prelingual deafness. On standardized tests, such as the Stanford Achievement Test, deaf students usually perform worst on vocabulary or word meaning subtests (King \& Quigley, 1985).

\section{Predicting the Effects of Variations in Word Exposure Frequencies}

Assuming that deafness limits exposure to ambient language, one or both of two effects of word exposure frequency might be observed in deaf adults' word familiarity ratings. First, individuals' ratings might shift uniformly (against the normative values) as a function of lifelong exposure frequency. This would be a scaling effect across the entire lexicon. For example, assuming the typical orientation of a familiarity rating scale, with numerically higher ratings equal to higher familiarity, individuals with lower exposure to language might give words uniformly lower ratings. Second, the ordering of words on the familiarity scale might differ for two individuals with different exposure frequencies. For example, an individual with greater exposure might tend to reflect a common, or population, probability distribution for words in the ambient language, whereas an individual with lower exposure might have idiosyncratic familiarity with words.

As a heuristic, a very simple sampling model $^{4}$ was used to predict the distribution of word frequencies that might be obtained by an individual with reduced word exposure. First, a 500-word pseudolexicon with frequencies distributed in a log-normal shape (Carroll, 1967; SPSS, 1997), as in the Kučera and Francis (1967) corpus, was generated. Word frequencies were converted to probabilities between zero and one, and those probabilities were arranged in order and were used to define probability ranges. To simulate lifelong word exposure, on a trial-bytrial basis, random numbers from a uniform distribution were generated and assigned to the appropriate probabil- 
ity ranges in the pseudolexicon. Thus, higher frequency words captured larger proportions of the randomly generated numbers than did lower frequency words. The total number of randomly generated numbers was varied to simulate two exposure groups (high, 50,000 numbers; low, 25,000 numbers), with 5 individuals in each group (i.e., five different sets of randomly generated numbers from the uniform distribution). ${ }^{5}$

First, to examine how the overall magnitude of familiarity ratings might rise or fall as a function of exposure, average frequencies of occurrence for the pseudolexicon were calculated. The pseudolexicon was subdivided into three word probability levels (high, medium, and low) to examine whether exposure resulted in a uniform scaling across high versus low exposure, independent of a word's frequency. Figure 1, which displays means as a function of word probability (high, medium, low) and exposure group, shows a simple scaling relationship between the exposure frequencies for the high and low groups. Second, the within- and between-group ordering of words on the familiarity scale was examined with within- and betweengroup correlations of simulated individual's item data. Figure 2 displays the mean correlation coefficients as a function of word probability and exposure group. The pattern of within- and between-group correlations was reduced in an orderly fashion as word exposure and probability declined.

\section{THE PRESENT STUDY}

The hypothesis for the present study was that if word familiarity ratings primarily index exposure to words in

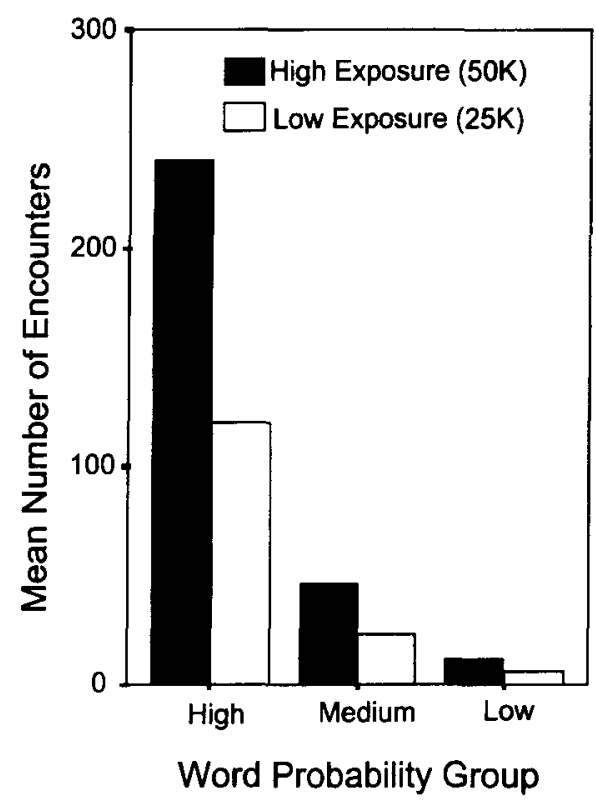

Figure 1. Mean simulated encounters are displayed as a function of word probability (high, medium, and low). Dark bars are means for the high-exposure simulations. Light bars are means for the low-exposure simulations.

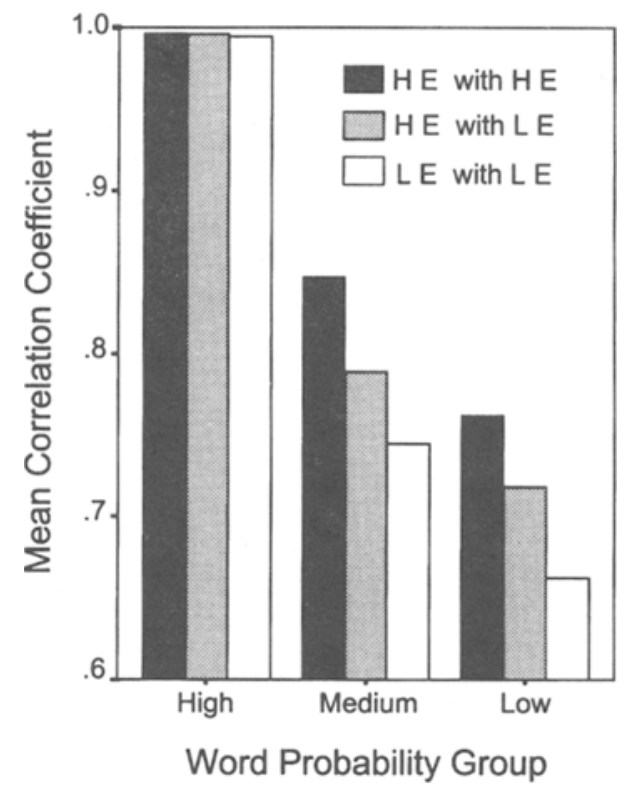

Figure 2. Mean Pearson correlation coefficients between individual simulated item encounter counts are displayed as a function of word probability group (high, medium, and low). Dark bars are the means of correlation coefficients for the highexposure (HE) simulations $(n=10)$. Gray bars are the means of correlation coefficients between the high- and low-exposure (LE) simulations $(n=25)$. Light bars are the means of correlation coefficients for the low-exposure simulations $(n=10)$.

the ambient linguistic environment, then the ratings obtained from these two populations should generally match the patterns of the simulations. First, mean familiarity should be lower for the deaf group than for the hearing group. This difference should simply reflect the application of a scaling factor across high-, medium-, and lowfamiliarity words. Second, the pattern of within- versus between-group correlations should be a reduction in the magnitude of correlation in an orderly fashion as a function of word frequency. Specifically, the highest intercorrelations should be among those individuals with the most exposure (hearing), and the lowest should be for the individuals with the least exposure (deaf).

\section{Method}

\section{Participants}

The participants were screened for the following characteristics: (1) between 18 and 45 years of age, (2) currently enrolled in or graduated from college, (3) no self-reported learning disabilities, (4) self-report of English as a native language, (5) vision 20/30 or better in each eye, as determined with a standard Snellen chart, and (6) average or better performance on a lipreading screening test, as referenced to the appropriate distribution of performance by deaf or hearing college-educated adults (Bernstein, Demorest, \& Tucker, 1997; Demorest \& Bernstein, 1992); additionally, deaf participants were screened to have (7) bilateral severe to profound hearing impairment (greater than $80 \mathrm{~dB}$ HL pure tone average across 500 , 1000 , and $2000 \mathrm{~Hz}$ ), (8) self-reported onset of loss prior to 4 years of age, (9) self-reported use of English as the primary language of the participant's family, and (10) education in mainstream and/or 
oral program for 8 or more years. All participants were paid for their participation.

Hearing participants. Sixty-four participants with normal hearing were recruited from among graduate students at Gallaudet University (GU) and from the campuses of California State University, Northridge (CSUN), University of Southern California, and University of California, Los Angeles. Seven House Ear Institute employees also participated. Thirteen participants were dropped due to technical malfunction, and 1 withdrew. Fourteen of the 50 participants in the resulting group were male, and 36 were female. Fifteen were from GU. The group mean age was 25.6 (range 18.5-43.8) years.

Deaf participants. Fifty-eight deaf participants were recruited from GU and CSUN. Eight were dropped due to a technical malfunction, early withdrawal from the study, or having been inappropriately selected based on review of their screening measures. Of the resulting 50,14 were from Gallaudet. Fourteen were male, and 36 were female. Participant-reported age at onset of hearing impairment was as follows: birth, $36 ; 0-1$ years, $4 ; 1-2$ years, $3 ; 2-3$ years, $2 ; 3-4$ years, 2 . Three participants reported an unknown age of onset; however, for all 3 , the hearing impairment was discovered prior to 4 years of age. The majority (43) of participants had $90 \mathrm{~dB}$ $\mathrm{HL}$ or greater pure tone averages (profound hearing impairment). The reported causes of the hearing impairments were as follows: unknown, 29; meningitis, 7; genetic/hereditary, 6; maternal rubella, 5 ; premature birth, 1; high fever, 1; other, 1 . The mean age across the group was 23 (range $18.0-32.0$ ) years.

\section{Materials and Procedure}

The data reported here were collected as part of a project to examine word lexical knowledge and processing in skilled deaf lipreaders. The project was begun at Gallaudet University (GU) and completed at the House Ear Institute (CA).

Reading and vocabulary tests. All of the participants were administered Form L of the Peabody Picture Vocabulary Test-Revised (PPVT; Dunn \& Dunn, 1981). For GU deaf participants, reading scores were obtained from the GU English Department. The CA deaf participants received the Advanced-2 Vocabulary and Reading Comprehension subtests of the Stanford Achievement Test-8th Edition (SAT-8; Psychological Corporation, 1989). The PPVT measures receptive vocabulary for Standard American English and is normed for spoken presentation. However, spoken presentation is inappropriate for deaf participants, because they can fail to perceive words correctly that they actually know. Therefore, words were presented orthographically on individually printed cards to deaf and hearing participants. The English Placement Test (EPT; Gallaudet English Department, 1976) was administered by the English Department to GU undergraduate students. On the reading comprehension subtest, students read short passages and then answered multiple-choice questions. Previous research at GU in Bernstein, Demorest, and Tucker (2000) showed a significant positive correlation ( $r$ values between .26 and .40 ) between reading (as measured with the GU EPT) and lipreading performance in deaf adults with English as a first language. The vocabulary and reading comprehension subtests of the SAT -8 (Psychological Corporation, 1989) were substituted for the EPT measures at the CA sites and were administered to all participants.

Lipreading screening test. The lipreading screening test comprised 30 videorecorded sentences (Bernstein \& Eberhardt, 1986) from the list of CID (Central Institute for the Deaf) Everyday Sentences (Davis \& Silverman, 1970). Half of the sentences were spoken by a male talker. Passing scores were based on normative data collected for the relevant participant group. The participants were tested individually in a quiet room. The prerecorded sentence stimuli were displayed on a 14-in. color monitor approximately $0.5 \mathrm{~m}$ from the participant. The participants viewed each sentence and then typed at a computer terminal what they thought the talker had said.

Word familiarity. The list of 450 words used by Lewellen et al. (1993) in a paper-and-pencil familiarity-rating task was obtained from the Speech Research Laboratory at Indiana University. The word list was selected from the pool of words previously rated for subjective familiarity on the Hoosier Mental Lexicon (HML) 7-point scale also employed in the present experiment (Nusbaum et al., 1984). On the basis of the Nusbaum et al. norms, the set of 450 stimulus words was broken into three subsets defined as low $(0-2.99$ in the HML, $n=150$ ), medium (3.00-5.0 in the HML, $n=151$ ), and high familiarity (5.01-7.00 in the HML, $n=149)$. An additional 173 words used in a preceding age-of-acquisition rating experiment (Auer, Waldstein, Tucker, \& Bernstein, 1996) were also rated but not evaluated here.

The participants were tested individually in a quiet room. They were seated in front of a computer monitor and were given verbal instructions. P.E.T. (coauthor), a certified sign language interpreter, administered instructions to the deaf participants using simultaneous communication (i.e., signs in English word order produced in synchrony with speech). Five different randomizations of the entire word list were generated. The participants were randomly assigned to randomizations of the familiarity word list, such that 10 participants in each participant group received the same randomization. Each randomized list was divided into two blocks. Each participant started with a 10 -word practice set of words, not part of the stimulus set, followed by the two test blocks.

In the familiarity judgment task, a trial began with the presentation of the word to be rated appearing in the center of the computer screen followed by a prompt to enter a familiarity rating. A 7-point familiarity scale was implemented on a labeled keyboard. The wording used to label the scale was based on the description provided in Nusbaum et al. (1984). ${ }^{6}$ The points on the scale were labeled as follows: $1=$ I've never seen, heard, or read the word before; 2 = I might have seen, heard, or read the word before; $3=$ $\Gamma$ ' $m$ pretty sure $\Gamma$ ve seen, heard, or read the word before; $4=\Gamma$ ve seen, heard, or read the word but don't know its meaning; $5=I$ 'm certain I've seen, heard, or read the word before but have only a vague idea of its meaning; $6=I$ think I know the meaning but $\Gamma \mathrm{m}$ not certain it's correct; $7=I$ know the word and am confident of its meaning. The participants could respond that they were familiar with part of the word; if they did so, they were prompted to type the part with which they were familiar. Immediately after a response was entered, the next word was presented.

\section{Results and Discussion \\ Descriptive Data for Participant Groups}

Reading and vocabulary tests. PPVT raw scores were transformed into standard score equivalents (SSEs), a measure of the deviation from the mean of the norming group. Figure 3 shows boxplots of the distributions of SSE scores. Boxes represent the interquartile ranges for each of the groups. Approximately $25 \%$ of the observations fall in the range defined by each whisker. A twoway analysis of variance (ANOVA) showed that groups (deaf vs. hearing) were different $[F(1,96)=41.69, p<$ $.001]$, but not the source of participants (GU vs. CA) nor the interaction. The deaf mean SSE was 89.9 , and the hearing mean SSE was 109.5. Sixteen deaf participants had SSEs of 100 or greater, and 34 participants were above 85 (one standard deviation below the standard mean). Thirty-eight hearing participants had SSEs of 100 or greater, and 49 participants were above 85 .

The deaf GU participants' EPT scores for both reading comprehension and vocabulary were strongly correlated with PPVT SSEs $(r=.894, n=12$; and $r=.924$, $n=10$, respectively). ${ }^{7}$ CA participants received the vocabulary and comprehension subtests of the SAT. The CA 


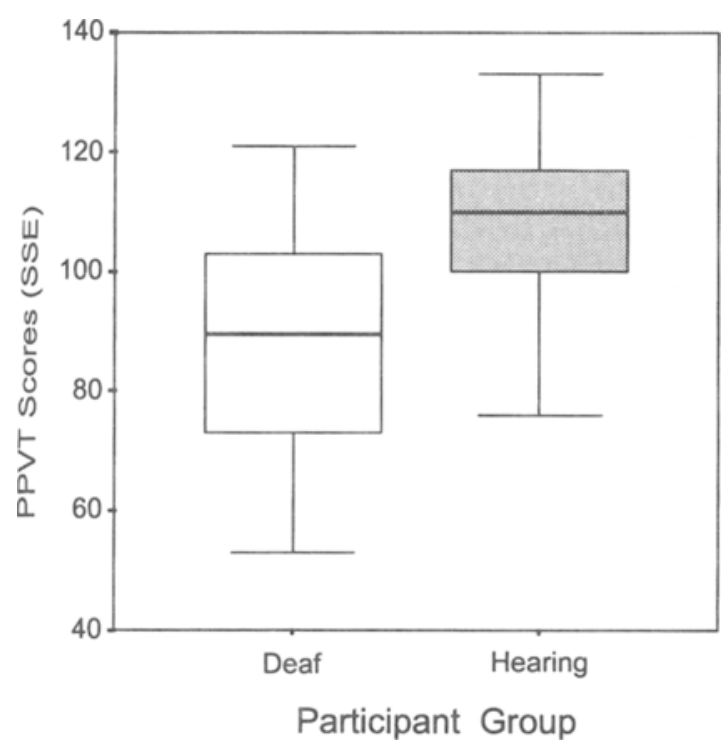

Figure 3. The distribution of PPVT SSE is displayed for each participant group. The distribution of the deaf participants' scores is on the left, and the distribution of the hearing participants' scores is on the right. The medians are marked with a horizontal line through each of the boxes, and horizontal lines (at the ends of the whiskers) mark the most extreme values not considered outliers (SPSS, 1996).

deaf participants scored significantly lower than the CA hearing participants on both the vocabulary subtest (deaf $M=77 \%$ correct vs. hearing $M=94 \%$ correct) $[t(69)=-4.966, p<.0001]$ and the comprehension subtest (deaf $M=71 \%$ correct vs. hearing $M=88 \%$ correct) $[t(69)=-5.124, p<.0001]$. For the CA deaf group, SAT comprehension and vocabulary scores were strongly correlated with PPVT scores (respectively, $r \mathrm{~s}=$ .805 and $.850, n=36$ ). For the CA hearing group, SAT comprehension and vocabulary scores were moderately correlated with PPVT scores (respectively, $r \mathrm{~s}=.686$ and $.630, n=35$ ). The lower correlations for the hearing participants were in part due to the high level of performance on the SAT subtests by the majority of participants (comprehension, $M=88 \%$ correct; vocabulary, $M=94 \%$ correct).

Taken together, the measures of reading comprehension and printed vocabulary knowledge provided a consistent description of the participants. The deaf group demonstrated poorer reading comprehension and poorer printed vocabularies than the hearing group. The conclusion that the two deaf groups were similar across locations is supported by both the strong correlations of comprehension and vocabulary measures with PPVT scores for both the CA and the GU deaf participants and the overlap of their distributions for several other descriptive measures. In contrast to the majority of early-onset profoundly deaf individuals, the present sample of deaf individuals had relatively high levels of reading comprehension and vocabulary knowledge.
Lipreading screening. After correcting unambiguous typographical errors, scoring of words correct in lipread sentences was performed by custom software. The deaf group mean score was $54.4 \%$ words correct, and the hearing group mean score was $28 \%$ words correct. All of the deaf participants scored above $75 \%$ of the hearing participants, and the best deaf participants far outperformed the best hearing participants. This between-group difference is not surprising, given the higher screening criterion used for the deaf participants. However, the betweengroup difference, particularly for the best performers in each participant group, was also expected on the basis of our previous research that demonstrated that the best lipreaders are deaf (Auer, Bernstein, \& Coulter, 1998; Bernstein et al., 1997, 2000).

\section{Subjective Familiarity Ratings}

Of the total 22,500 responses collected from each group, only a small percentage ( $3.2 \%$ by the deaf group, and $4.3 \%$ by the hearing group) of responses indicated familiarity with part of the word. Because the part-word responses were similar across groups and precluded the participant from entering a familiarity rating, those responses were not used in the analyses reported below.

An average familiarity rating for each subset of words (high, medium, and low HML familiarity) was calculated for each participant. Figure 4 shows the means of the familiarity ratings as a function of familiarity level and participant group. A $3 \times 2$ repeated measures ANOVA, with familiarity (high, medium, low) as a within-subjects factor and group (deaf vs. hearing) as a between-subjects factor, showed significant main effects of group $[F(1,98)=$

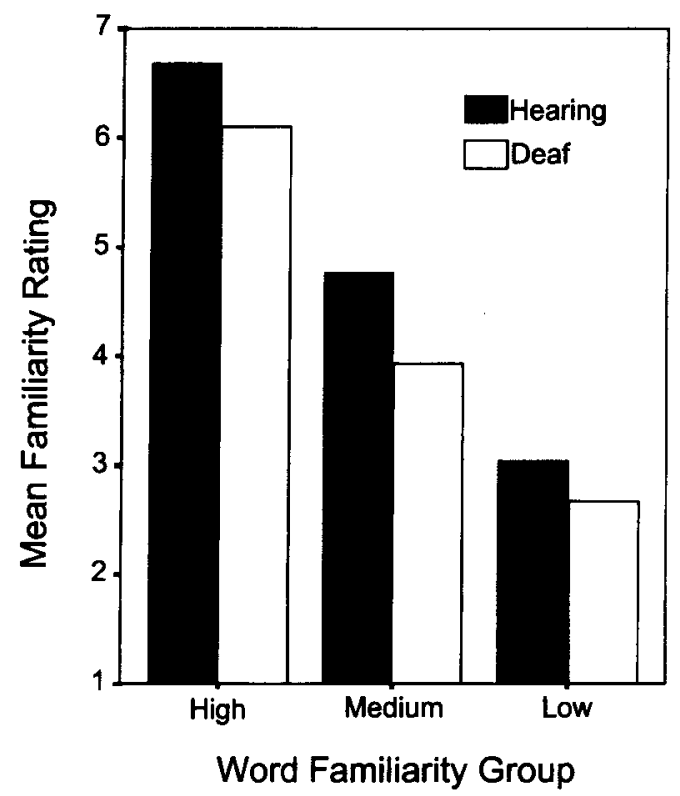

Figure 4. Mean familiarity ratings are displayed as a function of HML word familiarity group (high, medium, and low). Dark bars display the means for the hearing participants. Light bars display the means for the deaf participants. 
$15.95, p<.0001]$ and familiarity type $[F(2,196)=$ $1,515.92, p<.0001]$ and showed a significant interaction $[F(2,196)=6.26, p=.002]$. Obtained means were consistent with the HML ratings in Nusbaum et al. (1984). Consistent with the language measures presented above, on average, deaf participants rated words at each level to be less familiar than did hearing participants. The reduction in familiarity ratings was reasonably well predicted by a fixed ratio of .88 . The observed mean ratings by the deaf participants were as follows: 6.1 for the highfamiliarity set; 3.9 for the medium-familiarity set; and 2.7 for the low-familiarity set. The predicted values, computed by multiplying the mean observed ratings of hearing participants by .88 , were as follows: 5.9 for the highfamiliarity set; 4.2 for the medium-familiarity set; and 2.7 for the low familiarity set.

To examine the obtained orderings of words on the familiarity scale, item-level correlations were performed separately for high-, medium-, and low-familiarity levels. To reduce variability in the estimates, each participant group was split into 5 subgroups of 10 randomly selected participants. ${ }^{8}$ Within each subgroup, average familiarity ratings were calculated for each item. The mean item ratings for each subgroup were then correlated with those of the other 9 subgroups. At each familiarity level, the correlation coefficients were quite high (ranging from .51 to .86) both within and across participant groups. A $3 \times 3$ ANOVA, with familiarity level

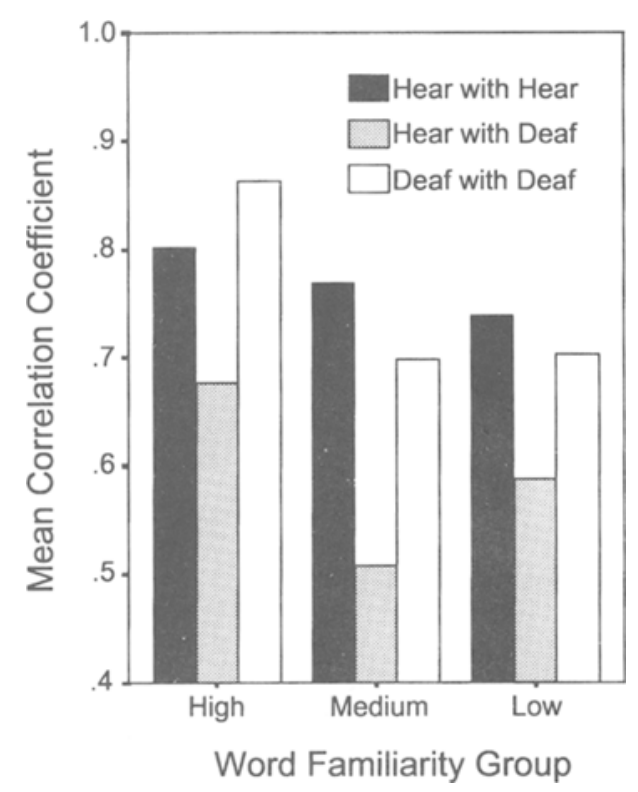

\footnotetext{
Figure 5. Mean Pearson correlation coefficients between participant subgroup item ratings are displayed as a function of HML word familiarity group (high, medium, and low). Dark bars display the mean correlation coefficients within the hearing participant subgroups $(n=10)$. Gray bars display the mean correlation coefficients between the hearing participant subgroups and deaf participant subgroups $(n=25)$. Light bars display the mean correlation coefficients within the deaf participant subgroups $(n=10)$.
}

(high, medium, low) and correlation type (hear-withhear, hear-with-deaf, deaf-with-deaf) as factors, showed significant main effects of correlation type $[F(2,134)=$ $97.19, p<.0001]$ and familiarity level $[F(2,134)=31.92$, $p<.0001]$ and showed a significant interaction $[F(4,134)=$ $4.90, p=.001]$. Figure 5 shows the mean correlations as a function of level and correlation type.

To investigate the interaction, one-way ANOVAs were performed for each familiarity level, with correlation type as the factor. Statistically significant main effects of correlation type were obtained at each familiarity level. Post hoc comparisons (Tukey, HSD) revealed that the betweenparticipant group correlations were significantly lower than the within-participant group correlations. That is, irrespective of the familiarity level, deaf participants ordered words on the familiarity scale more like other deaf participants than like hearing participants. Likewise, hearing participants ordered words on the familiarity scale more like other hearing participants than like deaf participants. This pattern was not predicted in the introduction, in which it was predicted that correlations would be lower among individuals with the least amount of experience. This result is discussed in detail in the General Discussion section.

Within-group average familiarity ratings were calculated for each stimulus item to compare with ratings obtained by Nusbaum et al. (1984). Correlations were performed on the item means from our two groups and the ratings in the HML (Nusbaum et al., 1984). The mean familiarity ratings for stimulus items were highly correlated across our groups $(r=.903)$, and average familiarity ratings of our groups were highly correlated with those in Nusbaum et al. ( $r=.875$ for the deaf group vs. HML; $r=.862$ for the hearing group vs. HML).

To assess the sensitivity of the familiarity ratings to within-group variation in language knowledge and ability, correlational analyses on familiarity ratings and reading scores were performed on individual participants' mean ratings within each group. Tables 1 and 2 display the Pearson correlation coefficients for each group. Mean familiarity ratings were significantly correlated with measures of printed language knowledge for each group. This is evidence that subjective familiarity ratings are sensitive to individual variation in language experience. The lack of a significant correlation for the descriptive language measures of the GU deaf participants may be due to the small sample (EPT vocabulary, $n=10$; EPT comprehension, $n=12$ ). The lipreading screening measure shown in the tables was not related to any other measures of language ability (cf. Bernstein et al., 1997; see also Geers \& Moog, 1989).

\section{GENERAL DISCUSSION}

In the present study, we obtained direct evidence for the sensitivity of word familiarity ratings to differences in language experience. The hearing participants had higher printed vocabulary and sentence comprehension 
Table 1

Pearson Correlation Coefficients for Individual Deaf Participants' Measures of Printed and Spoken Language

\begin{tabular}{|c|c|c|c|c|c|c|c|}
\hline \multirow[b]{2}{*}{ Measure } & \multirow{2}{*}{$\begin{array}{c}\text { Mean } \\
\text { Familiarity }\end{array}$} & \multicolumn{2}{|c|}{ SAT } & \multirow[b]{2}{*}{ PPVT } & \multirow[b]{2}{*}{ Lipreading } & \multicolumn{2}{|c|}{ EPT } \\
\hline & & Vocabulary & Comprehension & & & Vocabulary & Comprehension \\
\hline Mean Familiarity & - & $.49 * *$ & $.49^{* *}$ & $.49 * *$ & .03 & .38 & .53 \\
\hline \multicolumn{8}{|l|}{ SAT } \\
\hline Vocabulary & & - & $.85^{* *}$ & $.85^{* *}$ & .26 & d.u. & d.u. \\
\hline Comprehension & & & - & $.81^{* *}$ & .33 & d.u. & d.u. \\
\hline PPVT & & & & - & .20 & $.92 * *$ & $.89^{* *}$ \\
\hline Lipreading & & & & & - & .40 & .46 \\
\hline \multicolumn{8}{|l|}{ EPT } \\
\hline Vocabulary & & & & & & - & $.89 * *$ \\
\hline Comprehension & & & & & & & - \\
\hline
\end{tabular}

Note-Only CA deaf participants $(n=36)$ contributed to the correlations with the SAT scores. Only GU deaf participants $(n=14)$ contributed to the correlations with the EPT scores. d.u. = data unavailable for correlation. ${ }^{* *} p<.01$.

scores, and they consistently rated words as more familiar. Within groups, individuals' mean familiarity ratings were correlated with measures of printed language. More importantly, item-level correlations within deaf and hearing groups were higher than between-group correlations.

The results of the present experiment partially agree with the predictions made by the simple sampling model in the introduction for which it was assumed that deaf individuals differ from the hearing individuals only in quantity of word exposure. The prediction that reduction in familiarity for words spanning the range of familiarities can be scaled by a fixed ratio was confirmed. The prediction that consistency in word familiarity ordering is a function of exposure frequency was not. The difference in word familiarity orders between the groups is interpreted as evidence that deaf individuals have a different pattern of word experience than hearing individuals, in addition to their presumed lower word exposure.

What might account for differences in patterns of word experience across groups? First, reduced early exposure to a consistent source of ambient language could reduce exposure to specific parts of the lexicon. For example, certain words tend to be age specific in their usage (e.g., bunny, mommy, bow-wow), and these words may be omitted or underexposed for individuals with severe to profound hearing impairments. Second, difficulties in perceiving spoken language accurately may skew the frequency of experience for specific words. Most normal early language experience is through spoken communication. Because many words are visually ambiguous to the lipreader, perceived exposure rates may functionally vary, even if the ambient distribution of words is the same (Auer \& Bernstein, 1997). Third, the frequency distribution of words that our deaf participants were exposed to may be different due to differences in educational experience or some other nonperceptually based variable. For example, until the recent prevalence of closed captioning, deaf individuals had virtually no access to language on television, a word source of some (unspecified) importance to hearing individuals. Fourth, estimates of the vast number of word experiences - perhaps as many as 30 million exposures (Hart \& Risley, 1995) - by hearing children by the age of 3 years, a critical time for first language experience, may establish a particular structure for the mental lexicon throughout life, which is different when exposure rates are lower.

The significant relevant differences in the linguistic environments of our two participant groups remain an area for future research. A general question, whenever word frequency or familiarity is to be used as a factor in a psycholinguistic experiment, is how large the magnitude of difference in word experience between two populations must be to warrant the collection of new familiarity ratings.

Table 2

Pearson Correlation Coefficients for Individual Hearing Participants' Measures of Printed and Spoken Language

\begin{tabular}{|c|c|c|c|c|c|}
\hline \multirow[b]{2}{*}{ Measure } & \multirow{2}{*}{$\begin{array}{c}\text { Mean } \\
\text { Familiarity }\end{array}$} & \multicolumn{2}{|c|}{ SAT } & \multirow[b]{2}{*}{ PPVT } & \multirow[b]{2}{*}{ Lipreading } \\
\hline & & Vocabulary & Comprehension & & \\
\hline Mean & - & $.42^{*}$ & $.42^{*}$ & $.68^{* *}$ & -.17 \\
\hline \multicolumn{6}{|l|}{ SAT } \\
\hline Vocabulary & & - & $.49^{* *}$ & $.63^{* *}$ & -.10 \\
\hline Comprehension & & & - & $.69^{* *}$ & .01 \\
\hline PPVT & & & & - & .00 \\
\hline Lipreading & & & & & - \\
\hline
\end{tabular}

Note-Only CA hearing participants $(n=35)$ contributed to the correlations with the SAT scores. ${ }^{*} p<.05 . \quad{ }^{* *} p<.01$. 


\section{REFERENCES}

Akamatsu, C. T., \& Stewart, D. A. (1998). Constructing simultaneous communication: The contributions of natural sign language. Journal of Deaf Studies \& Deaf Education, 3, 302-319.

ALLEN, T. [E.] (1986). Patterns of academic achievement among hearing impaired students: 1974-1983. In A. N. Schildroth \& M. A Karchmer (Eds.), Deaf children in America (pp. 161-206). San Diego: College-Hill Press.

ALLEN, T. E. (1994). How many ASL users? Gallaudet University Center for Assessment and Demographic Studies [On-line]. Available: http://www.gallaudet.edu/ cadsweb/qxreasl.html

Amano, S., Kondo, T., \& KaKEHI, K. (1995). Modality dependency of familiarity ratings of Japanese words. Perception \& Psychophysics, 57, 598-603.

Auer, E. T., JR., \& Bernstein, L. E. (1997). Speechreading and the structure of the lexicon: Computationally modeling the effects of reduced phonetic distinctiveness on lexical uniqueness. Journal of the Acoustical Society of America, 102, 3704-3710.

Auer, E. T., JR., Bernstein, L. E., \& Coulter, D. C. (1998). Temporal and spatio-temporal vibrotactile displays for voice fundamental frequency: An initial evaluation of a new vibrotactile speech perception aid with normal-hearing and hearing-impaired individuals. Journal of the Acoustical Society of America, 104, 2477-2489.

Auer, E. T., Jr., Waldstein, R. S., Tucker, P. E., \& Bernstein, L. E. (1996). Relationships between word knowledge and visual speech perception: I. Subjective estimates of word age of acquisition [Abstract]. Journal of the Acoustical Society of America, 100, 2569

BaLOTA, D. A. (1994). Visual word recognition: The journey from features to meaning. In M. A. Gernsbacher (Ed.), Handbook of psycholinguistics (pp. 303-348). San Diego: Academic Press.

Balota, D. A., \& Chumbley, J. I. (1984). Are lexical decisions good measures of lexical access? The role of word frequency in the neglected decision stage. Journal of Experimental Psychology: Human Perception \& Performance, 10, 340-357.

Balota, D. A., Ferraro, F. R., \& Connor, L. T. (1991). On the early influence of meaning in word recognition: A review of the literature. In P. J. Schwanenflugel (Ed.), The psychology of word meanings (pp. 187-218). Hillsdale, NJ: Erlbaum

Bernstein, L. E., Demorest, M. E., \& Tucker, P. E. (1997). What makes a good lipreader? First you have to find one. In R. Cambell, B. Dodd, \& D. Burnham (Eds.), Hearing by eye II: Advances in the psychology of speechreading and auditory-visual speech (pp. 211227). East Essex, U.K.: Psychology Press.

Bernstein, L. E., Demorest, M. E., \& Tucker, P. E. (2000). Speech perception without hearing. Perception \& Psychophysics, 62, 233-252.

BERNSTEIN, L. E., \& EBERHARDT, S. P. (1986). Johns Hopkins lipreading corpus I-II: Disk 1 [Video disk]. Baltimore: Johns Hopkins University.

Brown, G. D. A., \& WATson, F. L. (1987). First in, first out: Word learning age and spoken word frequency as predictors of word familiarity and word naming latency. Memory \& Cognition, 15, 208-216.

Carroll, J. B. (1967). On sampling from a lognormal model of word frequency distribution. In H. Kučera \& W. N. Francis (Eds.), Computational analysis of present-day American English (pp. 406-424). Providence, RI: Brown University Press.

CARROLL, J. B. (1970). An alternative to Julliand's usage coefficient for lexical frequencies, and a proposal for a standard frequency index (SFI). Computer Studies in Humanities \& Verbal Behavior, 3, 61-65.

CARroll, J. B. (1971). Measurement properties of subjective magnitude estimates of word frequency. Journal of Verbal Learning \& Verbal Behavior, 10, 722-729.

Carrol.l, J. B., Davies, P., \& Richman, B. (1971). Word frequency book. New York: American Heritage.

Connine, C. M., Mullennix, J., Shernoff, E., \& Yelen, J. (1990). Word familiarity and frequency in visual and auditory word recognition. Journal of Experimental Psychology: Learning, Memory, \& Cognition, 16, 1084-1096.

CoRnett, O. (1967). Cued speech. American Annals of the Deaf, 112 , 3-13.

Davis, H., \& Silverman, S. R. (EDS.) (1970). Hearing and deafness (3rd ed.). New York: Holt, Rinehart \& Winston.
DEMOREST, M. E., \& BERNSTEIN, L. E. (1992). Sources of variability in speechreading sentences: A generalizability analysis. Journal of Speech \& Hearing Research, 35, 876-891.

DunN, L. M., \& DunN, L. M. (1981). Peabody Picture Vocabulary Test-Revised. Circle Pines, MN: American Guidance Service.

EUKEL, B. (1980). A phonotactic basis for word frequency effects: Implications for automatic speech recognition [Abstract]. Journal of the Acoustical Society of America, 68, S33.

Forster, K. I. (1976). Accessing the mental lexicon. In R. J. Wales \& E. Walker (Eds.), New approaches to language mechanisms (pp. 257284). Amsterdam: North-Holland.

Gallaudet English Department (1976). English Placement Test [Unpublished test]. Washington, DC: Gallaudet University.

GARBER, E. E., \& PISONI, D. B. (1991). Lexical memory in visual and auditory modalities: A second report (Research on Spoken Language Processing, Rep. No. PR-17). Bloomington: Indiana University.

GAYGEN, D. E., \& LUCE, P. A. (1998). Effects of modality on subjective frequency estimates and processing of spoken and printed words. Perception \& Psychophysics, 60, 465-483.

GeErs, A., \& MooG, J. (1989) Factors predicting the development of literacy in profoundly hearing-impaired adolescents. Volta Review, 91 , 69-86.

GerNSBACHER, M. A. (1984). Resolving 20 years of inconsistent interactions between lexical familiarity and orthography, concreteness, and polysemy. Journal of Experimental Psychology: General, 113, 256-281.

Gilhooly, K. J., \& LogIE, R. H. (1980). Age-of-acquisition, imagery, concreteness, familiarity, and ambiguity measures for 1,944 words. Behavior Research Methods \& Instrumentation, 12, 395-427.

GrISWOLD, L. E., \& Commings, J. (1974). The expressive vocabulary of preschool deaf children. American Annals of the Deaf, 119, 16-28.

HART, B., \& RISLEY, T. R. (1995). Meaningful differences in the everyday experience of young American children. Baltimore: Paul $\mathrm{H}$. Brookes.

Hirsh, K. W., \& Funnell, E. (1995). Those old, familiar things: Age of acquisition, familiarity and lexical access in progressive aphasia. Journal of Neurolinguistics, 9, 23-32.

Howes, D. H. (1957). On the relation between the intelligibility and frequency of occurrence of English words. Journal of the Acoustical Society of America, 29, 296-305.

KInG, C. M., \& Quigley, S. P. (1985). Reading and deafness. San Diego: College-Hill Press.

KuČERA, H., \& FrANCIS, W. N. (1967). Computational analysis of presentday American English. Providence, RI: Brown University Press.

LANDAuER, T. K., \& STREeTER, L. A. (1973). The structural differences between common and rare words: Failure of equivalence assumptions for theories of word recognition. Journal of Verbal Learning \& Verbal Behavior, 12, 119-131.

Lewellen, M. J., Goldinger, S. D., Pisoni, D. B., \& Greene, B. G. (1993). Lexical familiarity and processing efficiency: Individual differences in naming, lexical decision, and semantic categorization. Journal of Experimental Psychology: General, 122, 316-330.

LUCE, P. A., \& PISONI, D. B. (1998). Recognizing spoken words: The neighborhood activation model. Ear \& Hearing, 19, 1-36.

MorTON, J. (1969). The interaction of information in word recognition. Psychological Review, 76, 165-178.

Nusbaum, H. C., Pisoni, D. B., \& Davis, C. K. (1984). Sizing up the Hoosier Mental Lexicon: Measuring the familiarity of 20,000 words (Research on Spoken Language Processing, Rep. No. PR-10). Bloomington: Indiana University.

PITT, M. A., \& McQueEN, J. M. (1998). Is compensation for coarticulation mediated by the lexicon? Journal of Memory \& Language, 39 , 347-370.

Plaut, D. C., McClelland, J. L., Seidenberg, M. S., \& Patterson, K. (1996). Understanding normal and impaired word reading: Computational principles in quasi-regular domains. Psychological Review, 103, 56-115.

The Psychological Corporation (1989). Stanford Achievement Test Series, Eighth Edition. San Antonio, TX: Harcourt Brace Jovanovich.

RuBIN, D. C. (1976). Applying psychometric methods in linguistic research: Some recent advances. Linguistics, 168, 63-66.

SAVIN, H. B. (1963). Word-frequency effect and errors in the perception of speech. Journal of the Acoustical Society of America, 35, 200-206. 
SCHEIN, J. D. (1989). At home among strangers. Washington, DC: Gallaudet University Press.

SEIDENBERG, M. S. (1997). Language acquisition and use: Learning and applying probabilistic constraints. Science, 275, 1599-1603.

SeidenberG, M. S., \& MCClelland, J. L. (1989). A distributed, developmental model of word recognition and naming. Psychological Review, 96, 523-568.

Silverman-Dresner, T., \& Guilfoyle, G. R. (1972). Vocabulary norms for deaf children. Washington, DC: Alexander Graham Bell Association for the Deaf.

SPSS BASE7.5 FOR WINDOWS [Computer software]. (1997). SPSS Inc. Chicago: SPSS Inc.

THORNDIKE, E. L., \& LORGE, I. (1944). The teacher's word book of 30,000 words. New York: Columbia University, Teachers College Press.

Trybus, R., \& Karchmer, M. (1977). School achievement scores of hearing impaired children: National data on achievement status and growth patterns. American Annals of the Deaf, 122, 62-69.

Vitevitch, M. S., Luce, P. A., Charles-Luce, J., \& Kemmerer, D. (1997). Phonotactics and syllable stress: Implications for the processing of spoken nonsense words. Language \& Speech, 40, 47-62.

WALTER, G. G. (1978a). Lexical abilities of hearing and hearingimpaired children. American Annals of the Deaf, 123, 976-982.

WALTER, G. G. (1978b). Relationship of word knowledge to word frequency in young adult deaf students. Journal of Communication Disorders, 11, 137-148.

\section{NOTES}

1. Throughout this paper, the term deaf is applied to individuals with severe to profound hearing impairments $(80 \mathrm{~dB} \mathrm{HL}$ or greater threefrequency pure tone average in the better ear) who rely primarily on vision for speech perception. These individuals may use hearing aids and gain benefit from residual hearing, but their primary source for language acquisition is vision.

2. If parents are unaware of an infant's hearing loss, obviously interventions such as hearing aids and consistently facing the infant while talking will not occur. Infant hearing screening is helping to reduce the possibility that hearing loss in infants remains undetected. Progressive hearing impairments can also occur among infants, as well as sudden losses associated with illness. These too can result in delays in consistent language experience and difficulty in naming the exact time of hearing impairment onset. In our experiments, we attempt to obtain detailed information about history of hearing impairment progression, age of onset, and likelihood that, subsequent to the detection of the hearing impairment, appropriate intervention was administered. However, this type of retrospective evaluation is fraught with insurmountable pitfalls.

3. In this group, hearing aids are typically used to enhance lipreading, not the other way around.

4. The prediction made by the current sampling model should be consistent with those of connectionist learning models of language processing in which investigators have used samples of linguistic material (e.g., text corpora) to estimate the population probability distribution for encounters with various linguistic entities (e.g., words, syntactic structures) in the ambient linguistic environment (see Seidenberg \& McClelland, 1989, and Plaut et al., 1996, for an application of this technique to the modeling of the frequency effect in printed word recognition).

5. The simulations did not include a mechanism to model distortions that may arise from using a labeled familiarity scale. For example, the familiarity scale employed in the present study compresses the range for high-frequency words. This compression occurs because, if a word's meaning is known, it would be given the highest possible rating regardless of how frequently it is encountered. However, the choice to not include these effects in the modeling was not capricious, because the present study was designed to test the adequacy of word familiarity ratings for reflecting an individual's quantity of exposure.

6. In Nusbaum et al. (1984), a rating of 7 indicated that the word was familiar and its meaning was well known. A rating of 4 indicated the letter string was recognized as a word, but its meaning unknown. A rating of 1 indicated that the word was unknown.

7. Comprehension scores were not available for 2 of the GU participants, and vocabulary scores were not available for 4 of the GU participants, because these subtests had not been administered to these students.

8 . These same analyses were performed using individual participant data. The pattern of correlations was similar; however, the levels of all correlations were reduced substantially.

(Manuscript received November 17, 1998; revision accepted for publication October 11, 1999.) 